\title{
VOLUME-PRESERVING TRANSFORMATIONS IN SEVERAL COMPLEX VARIABLES ${ }^{1}$
}

\author{
ARNE MAGNUS
}

In this paper we study pseudo-conformal mappings, that is, transformations of four-dimensional continua by means of pairs of analytic functions, $u\left(z_{1}, z_{2}\right), v\left(z_{1}, z_{2}\right)$, of two complex variables $z_{1}, z_{2}$. In particular we study volume-preserving transformations, that is, mappings in which the volume of any four-dimensional domain $D$ in the $z_{1} z_{2}$ space equals the volume of the domain $D_{1}$ into which $D$ is carried by the mapping $\left(z_{1}, z_{2}\right) \rightarrow(u, v)$. As in the case of mappings by means of one complex variable, we adopt the convention that the volumes of regions which are covered by mappings more than once are counted with their multiplicities. It may be pointed out that the investigation of these maps constitutes a genuine "several variable" problem, since the corresponding maps associated with one complex variable reduce to trivial translations and rotations.

A particularly interesting subclass of the class of volume-preserving transformations is the class of mappings which are also univalent, that is, mappings which are one-to-one in both directions. Some families of such mappings will be exhibited.

The study of volume-preserving pseudo-conformal maps is suggested by the work of Bergman and others $[1]^{2}$ on pseudo-conformal maps of given domains which minimize the volume under certain conditions. Since the solutions of such minimum problems are determined only up to an arbitrary volume-preserving mapping, the investigation of the latter may prove to be useful in arriving at certain unique extremal mappings characterized by geometric properties.

It is easy to see that the volume $V_{1}$ of the pseudo-conformal image $D_{1}$ of $D$ is $[1$, p. 138]

$$
V_{1}=\iint_{D} \iint\left|\frac{\partial(u, v)}{\partial\left(z_{1}, z_{2}\right)}\right|^{2} d x_{1} d x_{2} d y_{1} d y_{2}, z_{j}=x_{j}+i y_{j}
$$

where

Presented to the Society, April 25, 1953; received by the editors May 15, 1953 and, in revised form, July 6, 1953.

1 Presented to the Graduate School of Washington University in partial fulfillment of the requirements for the degree of Doctor of Philosophy. The author is indebted to Professor Zeev Nehari for his encouragement and valuable advice.

2 Numbers in brackets refer to the references at the end of the paper. 


$$
\frac{\partial(u, v)}{\partial\left(z_{1}, z_{2}\right)}=u_{z_{1}} \cdot v_{z_{2}}-u_{z_{2}} \cdot v_{z_{1}}
$$

is the Jacobian of the transformation. Our problem thus reduces to the investigation of pairs of functions $(u, v)$ satisfying

$$
\left|\frac{\partial(u, v)}{\partial\left(z_{1}, z_{2}\right)}\right|=1 \text {. }
$$

Without loss of generality we may further restrict ourselves to the study of

$$
\frac{\partial(u, v)}{\partial\left(z_{1}, z_{2}\right)}=\left|\begin{array}{ll}
u_{z_{1}} & u_{z_{2}} \\
v_{z_{1}} & v_{z_{2}}
\end{array}\right|=1 .
$$

It is well known [3] that the general solution of the partial differential equation (1) can be represented in the form

$$
z_{1}=p+w_{q}, \quad z_{2}=q-w_{p}, \quad u=p-w_{q}, \quad v=q+w_{p},
$$

where $w=w(p, q)$ is an arbitrary function, and the parameters $p$ and $q$ are restricted by

$$
1+w_{p p} \cdot w_{q q}-\left(w_{p q}\right)^{2} \neq 0 .
$$

However, this form of the solution is not suitable for our present purposes.

The linear transformation

$$
\begin{aligned}
& u=\alpha z_{1}+\beta z_{2}, \\
& v=\gamma z_{1}+\delta z_{2},
\end{aligned} \quad\left|\begin{array}{cc}
\alpha & \beta \\
\gamma & \delta
\end{array}\right|=1
$$

satisfies (1); and, if $\left(u\left(z_{1}, z_{2}\right), v\left(z_{1}, z_{2}\right)\right)$ and $\left(U\left(z_{1}, z_{2}\right), V\left(z_{1}, z_{2}\right)\right)$ are two solutions of (1), then the composite transformation $(U(u, v)$, $V(u, v))$ is also a solution of (1). Combining these two results, we see that we may normalize any pair of solutions $(u, v)$ so that

$$
u=z_{1}+\text { higher powers of } z_{1} \text { and } z_{2}
$$

and

$$
v=z_{2}+\text { higher powers of } z_{1} \text { and } z_{2}
$$

are the Taylor expansions about $(0,0)$.

If $u$ and $v$ are polynomials, we may write

$$
u=\sum_{i=1}^{m} f_{i}\left(z_{1}, z_{2}\right), \quad v=\sum_{i=1}^{n} \phi_{i}\left(z_{1}, z_{2}\right)
$$


where the $f_{i}$ 's and $\phi_{i}$ 's are homogeneous polynomials of $i$ th degree. Inserting (2) in (1), we obtain

$$
1=\sum_{i, j=1}^{m, n}\left|\begin{array}{ll}
\frac{\partial f_{i}}{\partial z_{1}} & \frac{\partial f_{i}}{\partial z_{2}} \\
\frac{\partial \phi_{j}}{\partial z_{1}} & \frac{\partial \phi_{j}}{\partial z_{2}}
\end{array}\right|
$$

The Jacobian $\partial\left(f_{i}, \phi_{j}\right) / \partial\left(z_{1}, z_{2}\right)$ is a homogeneous polynomial of degree $i+j-2$. Comparing polynomials of the same degree of homogeneity on the right- and left-hand sides of (1a), we arrive at the following system of differential equations:

$$
\sum_{\mu=1}^{i}\left|\begin{array}{ll}
\frac{\partial f_{\mu}}{\partial z_{1}} & \frac{\partial f_{\mu}}{\partial z_{2}} \\
\frac{\partial \phi_{i+1-\mu}}{\partial z_{1}} & \frac{\partial \phi_{i+1-\mu}}{\partial z_{2}}
\end{array}\right|=\delta_{i, 1}, \quad i=1, \cdots, m+n-1 .
$$

In (3) we set $f_{\mu}=0$ and $\phi_{i+1-\mu}=0$ whenever the indices $\mu$ and $i+1-\mu$ lie outside the intervals $[1, m]$ and $[1, n]$, respectively.

Theorem 1. If $u=\sum_{i=1}^{m} f_{i}\left(z_{1}, z_{2}\right)$ and $v=\sum_{i-1}^{n} \phi_{i}\left(z_{1}, z_{2}\right)$, where the $f_{i}$ 's and $\phi_{i}$ 's are homogeneous polynomials of ith degree, satisfying the differential equation

$$
u_{z_{1}} \cdot v_{z_{2}}-u_{z_{2}} \cdot v_{z_{1}}=1
$$

then

(4) $f_{m-\mu}=\sum_{\gamma=0}^{\mu} c_{\gamma} \sum\left(\begin{array}{l}\frac{m-\gamma}{n} \\ \sum_{\alpha=1}^{n-1} \nu_{\gamma, \alpha}\end{array}\right) \frac{\left(\sum_{\alpha=1}^{n-1} \nu_{\gamma, \alpha}\right) !}{\prod_{\alpha=1}^{n-1}\left(\nu_{\gamma, \alpha} !\right)} \cdot \phi_{n}^{(m-\gamma) / n-\Sigma_{\alpha-1}^{n-1} p_{\gamma, \alpha}} \cdot \prod_{\alpha=1}^{n-1} \phi_{\alpha}^{n \gamma, \alpha}$ where $\mu=0,1, \cdots, m+n-3, f_{0}=f_{-1}=\cdots=0$, the $c_{\gamma}$ 's are constants, and the sum without limits is to be extended over all combinations of non-negative integral exponents $\nu_{\gamma, \alpha}$ satisfying

$$
\sum_{\alpha=1}^{n-1}(n-\alpha) \nu_{\gamma, \alpha}=\mu-\gamma .
$$

The proof of Theorem 1 depends partly upon the following lemma: If $g_{m-\lambda}$ and $\phi_{n}$ are two homogeneous functions in $z_{1}$ and $z_{2}$ of degree $m-\lambda$ and $n$, respectively, then $g_{m-\lambda}=F\left(\phi_{n}\right)$ implies 


$$
g_{m-\lambda}=c_{\lambda} \cdot \phi_{n}^{(m-\lambda) / n},
$$

$c_{\lambda}$ constant.

The proof of Theorem 1 proceeds by complete induction on $\mu$. The equations (3) are solved one by one starting with $i=m+n-1$, or

$$
\frac{\partial\left(f_{m}, \phi_{n}\right)}{\partial\left(z_{1}, z_{2}\right)}=0 .
$$

This gives $f_{m}=F\left(\phi_{n}\right)$ or, by the lemma,

$$
f_{m}=c_{0} \cdot \phi_{n}^{m / n},
$$

which agrees with (4).

Using (4) for $\mu=0, \cdots, \lambda-1$ we are now able to write equation (3) with $i=m+n-\lambda-1$ in the form

$$
\frac{\partial\left(f_{m-\lambda}-H\left(\phi_{n}, \cdots, \phi_{1}\right), \phi_{n}\right)}{\partial\left(z_{1}, z_{2}\right)}=0,
$$

where $H$ is the expression for $f_{m-\lambda}$ in (4) save for one term, $c_{\lambda} \cdot \phi_{n}^{(m-\lambda) / n}$. Thus

$$
f_{m-\lambda}-H\left(\phi_{n}, \cdots, \phi_{1}\right)=F\left(\phi_{n}\right)
$$

or, by the lemma,

$$
f_{m-\lambda}-H\left(\phi_{n}, \cdots, \phi_{1}\right)=c_{\lambda} \cdot \phi_{n}^{(m-\lambda) / n} .
$$

Thus the proof is complete when we show that the sum of Jacobians on the left-hand side of (3) can be written as the single Jacobian above. Performing the indicated differentiations in (3) and using the identities

$$
\left|\begin{array}{cc}
p\left(z_{1}, z_{2}\right) \frac{\partial \phi_{j}}{\partial z_{1}} & p\left(z_{1}, z_{2}\right) \frac{\partial \phi_{j}}{\partial z_{2}} \\
\frac{\partial \phi_{k}}{\partial z_{1}} & \frac{\partial \phi_{k}}{\partial z_{2}}
\end{array}\right|=-\left|\begin{array}{cc}
p\left(z_{1}, z_{2}\right) \frac{\partial \phi_{k}}{\partial z_{1}} & p\left(z_{1}, z_{2}\right) \frac{\partial \phi_{k}}{\partial z_{2}} \\
\frac{\partial \phi_{j}}{\partial z_{1}} & \frac{\partial \phi_{j}}{\partial z_{2}}
\end{array}\right|
$$

and

$$
p\left(z_{1}, z_{2}\right) \frac{\partial\left(\phi_{n}, \phi_{n}\right)}{\partial\left(z_{1}, z_{2}\right)}=\left|\begin{array}{cc}
p\left(z_{1}, z_{2}\right) \frac{\partial \phi_{n}}{\partial z_{1}} & p\left(z_{1}, z_{2}\right) \frac{\partial \phi_{n}}{\partial z_{2}} \\
\frac{\partial \phi_{n}}{\partial z_{1}} & \frac{\partial \phi_{n}}{\partial z_{2}}
\end{array}\right|=0,
$$

we may write the left-hand side of (3) as the sum of two kinds of 
determinants. Those of the first kind are of the form

$$
-\left|\begin{array}{cc}
p\left(z_{1}, z_{2}\right) \frac{\partial \phi_{j}}{\partial z_{1}} & p\left(z_{1}, z_{2}\right) \frac{\partial \phi_{j}}{\partial z_{2}} \\
\frac{\partial \phi_{n}}{\partial z_{1}} & \frac{\partial \phi_{n}}{\partial z_{2}}
\end{array}\right| .
$$

Grouping these determinants properly and adding a proper multiple of

$$
\frac{\partial\left(\phi_{n}, \phi_{n}\right)}{\partial\left(z_{1}, z_{2}\right)}
$$

we obtain

$$
\frac{\partial\left(f_{m-\lambda}, \phi_{n}\right)}{\partial\left(z_{1}, z_{2}\right)}-\frac{\partial\left(H, \phi_{n}\right)}{\partial\left(z_{1}, z_{2}\right)} .
$$

The determinants of the second kind are of the form

$$
\left|\begin{array}{cc}
p\left(z_{1}, z_{2}\right) \frac{\partial \phi_{j}}{\partial z_{1}} & p\left(z_{1}, z_{2}\right) \frac{\partial \phi_{j}}{\partial z_{2}} \\
\frac{\partial \phi_{k}}{\partial z_{1}} & \frac{\partial \phi_{k}}{\partial z_{2}}
\end{array}\right|
$$

where both $j$ and $k$ are different from $n$. These determinants however cancel each other in pairs.

In order to establish that all determinants that appear in the proof (and no others) really are present, it is necessary to apply (5) for various values of $\mu$ (and $\gamma$ ). We may establish the needed inequalities for the subscripts $\alpha$ of the $\phi_{\alpha}$ as follows: If $\nu_{\gamma, \beta} \geqq 1$, then

$$
n-\beta \leqq(n-\beta) \nu_{\gamma, \beta} \leqq \sum_{\alpha=1}^{n-1}(n-\alpha) \nu_{\gamma, \alpha}=\mu-\gamma,
$$

or

$$
n-\beta \leqq \mu-\gamma .
$$

The proof of Theorem 1 depends on the homogeneity of $f_{m-\mu}$ and $\phi_{\nu}$, but not directly on the fact that these functions are polynomials. One may, therefore, extend the above method to a wider class of functions than pairs of polynomials.

For $m \leqq \mu \leqq m+n-3$ we have $f_{m-\mu}=0$, so that (4) is then an equation between the $\phi_{\nu}$ 's alone. We may obtain another formulation 
of Theorem 1 as follows. We first write $v$ binomially as

$$
v=\phi_{n}+\sum_{\alpha=1}^{n-1} \phi_{\alpha}
$$

and expand $v^{(m-\gamma) / n}$ in the binomial series

$$
\begin{aligned}
v^{(m-\gamma) / n} & =\sum_{s \gamma=0}^{\infty}\left(\begin{array}{c}
(m-\gamma) / n \\
s_{\gamma}
\end{array}\right) \phi_{n}^{(m-\gamma) / n-8 \gamma}\left(\phi_{n-1}+\cdots+\phi_{1}\right)^{s \gamma} \\
& =\sum_{s \gamma=0}^{\infty} \sum^{\prime}\left(\begin{array}{c}
(m-\gamma) / n \\
s_{\gamma}
\end{array}\right) \frac{s_{\gamma} !}{\prod_{\alpha=1}^{n-1}\left(\nu_{\gamma, \alpha} !\right)} \phi_{n}^{(m-\gamma) / n-s_{\gamma}} \prod_{\alpha=1}^{n-1} \phi_{\alpha}^{\gamma_{\gamma}, \alpha},
\end{aligned}
$$

where the sum $\sum^{\prime}$ is to be extended over all combinations of nonnegative exponents $\nu_{\gamma, \alpha}$ satisfying

$$
\sum_{\alpha=1}^{n-1} \nu_{\gamma, \alpha}=s_{\gamma}
$$

The function $\phi_{n}^{m-\gamma / n-s_{\gamma}} \prod_{\alpha=1}^{n-1} \phi_{\alpha}^{\gamma \gamma, \alpha}$ is homogeneous of degree

$$
m-\gamma-n s_{\gamma}+\sum_{\alpha=1}^{n-1} \alpha \cdot \nu_{\gamma, \alpha}=m-\gamma-\sum_{\alpha=1}^{n-1}(n-\alpha) \nu_{\gamma, \alpha} .
$$

Thus, for terms in (6) of degree $m-\mu$, we have

$$
\sum_{\alpha=1}^{n-1}(n-\alpha) \nu_{\gamma, \alpha}=\mu-\gamma,
$$

which shows that the sum of the terms in (6) of degree of homogeneity $m-\mu$ is exactly the coefficient of $c_{\gamma}$ in (4). Therefore, the sum of all terms of degree of homogeneity $\geqq 3-n$ in

$$
\sum_{\gamma=0}^{m+n-3} c_{\gamma} \cdot v^{(m-\gamma) / n}
$$

equals

$$
\sum_{\mu=0}^{m+n-8} f_{m-\mu}
$$

The sum (8) equals $u$ for $n \geqq 2$. For $n=1$, we have

$$
u=\alpha z_{1}+\beta z_{2}+\sum_{\mu=2}^{m} c_{\mu}\left(\gamma z_{1}+\delta z_{2}\right)^{\mu}, \quad v=\gamma z_{1}+\delta z_{2},
$$

where 


$$
\left|\begin{array}{ll}
\alpha & \beta \\
\gamma & \delta
\end{array}\right|=1
$$

The multiple series (6) converges when $\left|\phi_{n}\right|^{-1} \cdot \sum_{\alpha=1}^{n-1}\left|\phi_{\alpha}\right|<1$, that is, when

$$
r^{-n} \sum_{\alpha=1}^{n-1}\left|\phi_{\alpha}\right|<r^{-n}\left|\phi_{n}\right|,
$$

setting $r=\left[\left|z_{1}\right|^{2}+\left|z_{2}\right|^{2}\right]^{1 / 2}$. If we let $\left(z_{1}, z_{2}\right) \rightarrow(\infty, \infty)$, we may show: 1. $r^{-n}\left|v-\phi_{n}\right| \leqq r^{-n} \sum_{\alpha=1}^{n-1}\left|\phi_{\alpha}\right| \rightarrow 0 ; 2$. (9) is fulfilled if $r^{-n}\left|\phi_{n}\right|>\epsilon>0$; 3. $r^{-n}|v| \rightarrow 0$ if and only if $r^{-n}\left|\phi_{n}\right| \rightarrow 0$. Combining these results with (7) and (8), we obtain another formulation of Theorem 1:

Theorem 2. If $u$ and $v$ are two polynomials of degrees $m$ and $n$ $(m \geqq n)$, respectively, in the complex variables $z_{1}$ and $z_{2}$, which satisfy

$$
u_{z_{1}} \cdot v_{z_{2}}-u_{z_{2}} \cdot v_{z_{1}}=1
$$

then there exist constants $c_{0}, \cdots, c_{m+n-3}$, and to every $\epsilon>0$ there exists a constant $M=M(\epsilon)$, such that

$$
\left|z_{1}\right|>M,\left|z_{2}\right|>M \quad \text { and }\left[\left|z_{1}\right|^{2}+\left|z_{2}\right|^{2}\right]^{-n / 2} \cdot|v|>\epsilon
$$

implies

$$
\left|u-\sum_{\gamma=0}^{m+n-3} c_{\gamma} \cdot v^{(m-\gamma) / n}\right|<M \cdot\left[\left|z_{1}\right|^{2}+\left|z_{2}\right|^{2}\right]^{(-n+2) / 2} .
$$

We note that the zeros of $\phi_{n}$ lie on $n$ two-dimensional planes through $(0,0)$ and are therefore determined by $n$ complex numbers $z_{1} \cdot z_{2}^{-1}=k_{i}, i=1, \cdots, n$, where some or all $k_{i}$ may be $\infty$. We may easily prove that $r^{-n}\left|\phi_{n}\right| \rightarrow 0$ if and only if $z_{1} \cdot z_{2}^{-1}$ approaches some $k_{i}$. Thus the condition $\left[\left|z_{1}\right|^{2}+\left|z_{2}\right|^{2}\right]^{-n / 2} \cdot|v|>\epsilon>0$ as $\left(z_{1}, z_{2}\right) \rightarrow(\infty, \infty)$ is satisfied for all values of $z_{1} \cdot z_{2}^{-1}$ except in a neighborhood of each $k_{i}$.

We shall now apply Theorem 1 to determine all maps in which $n=2$ or $n=3$.

Theorem 3. If $u$ and $v$ are polynomials of degrees $m$ and $n(m \geqq n)$, respectively, satisfying the equation

$$
u_{z_{1}} \cdot v_{z_{2}}-u_{z_{2}} \cdot v_{z_{1}}=1,
$$

then $n \mid m$ for $n=2$ or $n=3$.

This means that $u=u_{r}$ and $v=v_{r}$ may be built up from the solution $u_{1}=\alpha z_{1}+\beta z_{2}, v_{1}=\gamma z_{1}+\delta z_{2}$ of (1) through successive solutions $u_{\nu}, v_{\nu}$ $(\nu=1, \cdots, r)$ of (1) by means of the constructions $u_{v+1}=u_{v}, v_{\nu+1}$ 
$=v_{\nu}+P\left(u_{v}\right)$; or $u_{v+1}=u_{\nu}+P\left(v_{v}\right), v_{v+1}=v_{\nu}$, where $P$ is a polynomial. For, if $n \mid m$, then $f_{m}=c_{0} \cdot \phi_{n}^{m / n}$, so that

$$
u_{r-1}=u_{r}-c_{0} \cdot v^{m / n}, \quad v_{r-1}=v_{r}
$$

is again a solution of (1) where the degree $m_{r-1}$ of $u_{r-1}$ is less than $m$. Repeating this process until the degree $m_{\nu}$ of $u_{\nu}$ is less than $n$, we see that $m_{\nu}=1$ and $\nu=2$. Thus

$$
u_{2}=\alpha z_{1}+\beta z_{2}, \quad v_{2}=\gamma z_{1}+\delta z_{2}+A\left(\alpha z_{1}+\beta z_{2}\right)^{2}+B\left(\alpha z_{1}+\beta z_{2}\right)^{3}
$$

and $u_{r}=u_{3}=u_{2}+P\left(v_{2}\right), v_{r}=v_{3}=v_{2}$ where $P$ is a polynomial.

To prove Theorem 3, we first consider $n=2$. We assume $m$ is odd. Then $f_{m}=c_{0} \cdot \phi_{2}^{m / 2}=c_{0}\left(\alpha z_{1}+\beta z_{2}\right)^{m}$ and $\phi_{2}=\left(\alpha z_{1}+\beta z_{2}\right)^{2}=h^{2}$. Inserting the expression for $\phi_{2}$ in (4), we see that the exponent of $h$ is

$$
E=m-\gamma-2 \nu_{\gamma, 1}=m-\gamma-2(\mu-\gamma)=m-2 \mu+\gamma \geqq m-2 \mu .
$$

Thus $E_{\min }=m-2 \mu$, the minimum of $E$, is attained only when $\gamma=0$ and $\nu_{0,1}=\mu$. The corresponding term in (4) is

$$
c_{0}\left(\begin{array}{c}
m / 2 \\
\mu
\end{array}\right) h^{m-2 \mu} \cdot \phi_{1}^{\mu} \text {. }
$$

We choose $\mu$ so that $m-2 \mu<0$, multiply (4) by $h^{2 \mu-m-1}$, and see that $h \mid \phi_{1}=z_{2}$ for normalized solutions. Thus $h=\beta z_{2}$ and $\phi_{2}=\beta^{2} z_{2}^{2}$. Inserting $\phi_{1}=z_{2}$ and $\phi_{2}=\beta^{2} z_{2}^{2}$ in (4) with $\mu=m-1$, we obtain

$$
z_{1}=f_{1}=\sum \text { polynomials in } z_{2} \text {, }
$$

a contradiction. Thus $2 \mid m$. In particular, $n=2$ and $m=3$ is impossible. Next we consider $n=3, m \neq 0(\bmod 3)$. Since $(m, 3)=1, f_{m}$ $=c_{0} \cdot \phi_{3}^{m / 3}$ implies $\phi_{3}=h^{3}$. The exponent $E$ of $h$ is then

$$
E=m-\gamma-3\left(\nu_{\gamma, 1}+\nu_{\gamma, 2}\right),
$$

subject to the restriction

$$
2 \nu_{\gamma, 1}+\nu_{\gamma, 2}=\mu-\gamma .
$$

Solving for $\nu_{\gamma, 2}$ and inserting in $E$, we obtain

$$
E=m-3 \mu+2 \gamma+3 \nu_{\gamma, 1} \geqq m-3 \mu .
$$

The minimum $E_{\min }=m-3 \mu$ is attained only for $\gamma=0, \nu_{0,1}=0$ and $\nu_{0,2}=\mu$. The corresponding term in (4) is

$$
c_{0}\left(\begin{array}{c}
m / 3 \\
\mu
\end{array}\right) h^{m-3 \mu} \cdot \phi_{2}^{\mu} \text {. }
$$

Again we choose $\mu$ so that $m-3 \mu<0$, and obtain 


$$
h \mid \phi_{2}, \quad \phi_{2}=h \cdot \eta_{1} .
$$

Repeating this argument twice, we first see that $h^{2} \mid \phi_{2}$ and next (restricting ourselves to even values of $\mu$ ) that $h \mid \phi_{1}$. Thus $h=\beta z_{2}$ and $z_{1}=f_{1}=\sum$ polynomials in $z_{2}$, which is the desired contradiction. One may prove in general by an extension of this method that $(n, m)=1$ and that $m=6, n=4$ are impossible. It therefore seems reasonable to conjecture that all polynomial solutions of (1) may be built up from $u_{1}=z_{1}, v_{1}=z_{2}$ in the manner described following Theorem 3 , or that $n \mid m$ or $m \mid n$.

Leaving the polynomial solutions of (1), it is easily seen that

$$
\begin{array}{ll}
u=z_{1}+\frac{1}{\alpha} \cdot F\left(\alpha z_{1}+\beta z_{2}\right) & \\
v=z_{2}-\frac{1}{\beta} \cdot F\left(\alpha z_{1}+\beta z_{2}\right), & \alpha \beta \neq 0,
\end{array}
$$

where $F$ is an entire function, is a solution of (1). Here

$$
\alpha u+\beta v=\alpha z_{1}+\beta z_{2}
$$

which makes it evident that the map (10) is univalent. By (11) any member of the family of the parallel analytic planes

$$
\alpha z_{1}+\beta z_{2}=\gamma \quad(\gamma \text { complex constant })
$$

is translated into itself by the vector $((1 / \alpha) F(\gamma),-(1 / \beta) F(\gamma))$. Other families of univalent volume-preserving maps are

$$
\begin{aligned}
& u=z_{1}+F\left(z_{2}\right), \quad v=z_{2}, \\
& u=z_{1}, \quad v=z_{2}+F\left(z_{1}\right) ;
\end{aligned}
$$

and

$$
u=z_{1} \cdot e^{F\left(z_{1} \cdot z_{2}\right)}, \quad v=z_{2} \cdot e^{-F\left(z_{1} \cdot z_{2}\right)},
$$

with $u v=z_{1} z_{2}$. Thus the analytic surfaces

$$
z_{1} \cdot z_{2}=\gamma, \quad \gamma \text { complex constant, }
$$

are invariant under the map (12).

To extend (10) and (12) to $n$ variables, we choose $\alpha_{1}, \cdots, \alpha_{n}$ and $\lambda_{1}, \cdots, \lambda_{n}$ so that

$$
\sum_{\nu=1}^{n} \lambda_{\nu}=0, \quad \prod_{\nu=1}^{n} \alpha_{\nu} \neq 0
$$

and set 


$$
u_{i}=z_{i}+\frac{\lambda_{i}}{\alpha_{i}} F\left(\sum_{\nu=1}^{n} \alpha_{\nu} z_{\nu}\right), \quad i=1, \cdots, n
$$

where

$$
\sum_{\nu=1}^{n} \alpha_{\nu} z_{\nu}=\gamma, \quad \gamma \text { complex constant, }
$$

are the invariant $2 n-2$ dimensional hyperplanes. In

$$
u_{i}=z_{i} \cdot e^{\lambda_{i} F\left(\Pi_{\nu=1}^{n} z_{\nu}\right)}, \quad i=1, \cdots, n,
$$

the $2 n-2$ dimensional surfaces

$$
\prod_{\nu=1}^{n} z_{\nu}=\gamma, \quad \gamma \text { complex constant, }
$$

are invariant. Both $(10 n)$ and $(12 n)$ are invariant volume-preserving transformations satisfying

$$
\frac{\partial\left(u_{1}, \cdots, u_{n}\right)}{\partial\left(z_{1}, \cdots, z_{n}\right)}=1 .
$$

The map (12) is suggested by the addition formula for $e^{z}$. Applying the addition formulas for $e^{n z}$ and $\tan z$ in a similar manner, we obtain the following univalent but not volume-preserving maps:

$$
\begin{aligned}
& u=z_{1} \cdot e^{(1 / n) F\left(z_{1}^{n} \cdot z^{m}\right)} \\
& v=z_{2} \cdot e^{-(1 / m) F\left(z^{n} \cdot z_{2}^{m}\right)}, \quad m, n \text { positive integers, } \\
& 12^{2}
\end{aligned}
$$

and

$$
u=\frac{z_{1}+F\left(\frac{z_{1}-z_{2}}{1+z_{1} z_{2}}\right)}{1-z_{1} F\left(\frac{z_{1}-z_{2}}{1+z_{1} z_{2}}\right)}, \quad v=\frac{z_{2}+F\left(\frac{z_{1}-z_{2}}{1+z_{1} z_{2}}\right)}{1-z_{2} F\left(\frac{z_{1}-z_{2}}{1+z_{1} z_{2}}\right)}
$$

where $F$ is an entire function. The invariant surfaces of (13) and (14) are given by

$$
\begin{gathered}
z_{1}^{n} \cdot z_{2}^{m}=\gamma, \\
\frac{z_{1}-z_{2}}{1+z_{1} z_{2}}=\gamma,
\end{gathered}
$$

respectively. The domain of definition of (14) must be restricted so that no denominator can be zero. 


\title{
REFERENCES
}

1. S. Bergman, The kernel function and conformal mapping, Mathematical Surveys, no. 5, American Mathematical Society, New York, 1950.

2. S. Bochner and W. T. Martin, Several complex variables, Princeton University Press, 1948.

3. G. Scheffers, Math. Zeit. vol. 2, p. 181.

[1] and [2] contain extensive bibilographies.

WASHINGTON UNIVERSITY

\section{A PROPERTY OF QUASI-CONFORMAL MAPPING}

\author{
H. L. ROYDEN
}

Let $W_{1}$ and $W_{2}$ be two open Riemann surfaces such that there is a quasi-conformal [1] mapping $h$ of $W_{1}$ onto $W_{2}$. Then it is known [2] that either both $W_{1}$ and $W_{2}$ have Green's function or else neither has, i.e., quasi-conformal mapping preserves the class $O_{G}$ of those surfaces which have no Green's function. From this one is led to the conjecture that quasi-conformal mapping preserves the classes $O_{H B}$ and $O_{H D}$ of surfaces which have no bounded harmonic function or harmonic functions with a finite Dirichlet integral, respectively. In the present paper we shall show that the ring $H D$ of bounded harmonic functions with a finite Dirichlet integral is the same for both $W_{1}$ and $W_{2}$. This has as a consequence not only the result of Pfluger on the preservation of the class $O_{G}$ but also the preservation of the class $O_{H D}$ under quasi-conformal mapping.

Whether or not $O_{H B}$ is preserved under quasi-conformal mapping is an open question. Another interesting open question is whether or not two topologically equivalent surfaces which have the same ring $H B D$ defined on them admit of a quasi-conformal mapping from one to the other.

1. Some rings of functions. Let $B D=B D_{i}$ be the ring of all piecewise smooth functions defined on the Riemann surface $W=W_{i}$ which are bounded and have a finite Dirichlet integral. A topology is introduced in $B D$ by defining

$$
f_{,} \rightarrow f
$$

if $\left|f_{\nu}\right|$ is uniformly bounded, $f$ converges to $f$ uniformly on every

Received by the editors July 27, 1953. 